\title{
Long-term survival in Japanese renal transplant recipients with Alport syndrome: a retrospective study
}

\author{
Ai Katsuma1, Yasuyuki Nakada', Izumi Yamamoto ${ }^{1 *}$ (D), Shigeru Horita ${ }^{2}$, Miyuki Furusawa ${ }^{3}$, Kohei Unagami ${ }^{3}$, \\ Haruki Katsumata ${ }^{1}$, Masayoshi Okumi ${ }^{3}$, Hideki Ishida ${ }^{3}$, Takashi Yokoo ${ }^{1}$, Kazunari Tanabe ${ }^{3}$ and Japan Academic \\ Consortium of Kidney Transplantation (JACK)
}

\begin{abstract}
Background: Patients with Alport syndrome (AS) develop progressive kidney dysfunction due to a hereditary type IV collagen deficiency. Survival of the kidney allograft in patients with AS is reportedly excellent because AS does not recur. However, several studies have implied that the type IV collagen in the GBM originates from podocytes recruited from the recipient's bone marrow-derived cells, suggesting the possibility of AS recurrence. Limited data are available regarding AS recurrence and graft survival in the Japanese population; the vast majority were obtained from living related kidney transplantation (LRKTX).

Methods: In this retrospective study, twenty-one patients with AS were compared with 41 matched patients without AS from 1984 to 2015 at two centers using propensity scores. Nineteen of the 21 patients with AS underwent LRKTx. The mean post-transplant follow-up period was 83 months in the AS group and 110 months in the control group. Histopathological AS recurrence was assessed by immunoreactivity of a5 (type IV collagen) antibody and electron microscopy.

Results: The graft survival rate was equivalent between patients with and without AS (86.7\% vs. $77.1 \%$ and $69.3 \%$ vs. $64.2 \%$ at 5 and 10 years; $p=0.16$, log-rank test). Immunoreactivity to a5 antibody showed strong linear positivity with no focal defect in six patients. Electron microscopy showed no GBM abnormalities in two patients who were exhibiting long-term kidney allograft survival.

Conclusions: We confirmed that a5 and the GBM structure were histopathologically maintained in the long term after kidney transplantation. The patient and graft survival rates were equivalent between Japanese patients with and without AS.
\end{abstract}

Keywords: Alport syndrome, Kidney transplantation, Type IV collagen

\section{Background}

Alport syndrome (AS) is an inherited nephropathy characterized by sensorineural hearing loss and typical ocular abnormalities. It is caused by a hereditary type IV collagen deficiency [1-3]. In $85 \%$ of patients with AS, the collagen deficiency exhibits X-linked inheritance (COL4A5 gene), and the remaining $15 \%$ show autosomal recessive and

\footnotetext{
* Correspondence: izumi26@jikei.ac.jp

${ }^{1}$ Division of Nephrology and Hypertension, Department of Internal Medicine, The Jikei University School of Medicine, 3-25-8, Nishi-Shimbashi, Minato-ku, Tokyo 105-8461, Japan

Full list of author information is available at the end of the article
}

rarely autosomal dominant inheritance (COL4A3 or COL4A4 genes). Histopathologically, these genetic alterations reflect glomerular basement membrane (GBM) thickening and lamellation, leading to focal segmental glomerulosclerosis and resultant global sclerosis and/or hyalinosis [1]. With respect to therapy, several studies have demonstrated the efficacy of angiotensin-converting enzyme inhibitors as the first-choice medications $[4,5]$, with angiotensin II receptor blockers and spironolactone as alternatives [6]. However, these medications are not always satisfactory, and there are no disease-specific medications. For these reasons, AS commonly progresses gradually 
from childhood, resulting in end-stage renal disease at a young age [3]. Indeed, according to the United States Renal Data System, the median age at which renal replacement therapy was initiated in AS from 2005 to 2009 was 33.7 years [7].

Patients with AS account for $>1 \%$ of all patients undergoing kidney transplantation [8]. Kidney allograft survival in patients with AS is excellent despite the fact that AS recipients can develop anti-GBM disease, which can induce allograft loss at a high rate early after kidney transplantation. These excellent results have been reported in several countries. These reasons were mainly explained by the lack of risk factors for chronic kidney disease (hypertension and diabetes mellitus), the absence of AS recurrence, and the very low rate of anti-GBM disease (only 1.9\%) [9]. However, the clinical outcomes of renal allografts in the Japanese population, the vast majority of whom have undergone living related kidney transplantation (LRKTx), have not yet been investigated.

Another aim of this study was to evaluate the recurrence of AS in recipients of kidney transplantation. This topic was investigated for the following three reasons. First, the type IV collagen $\alpha 3 \alpha 4 \alpha 5$ heterotrimer in the GBM originates only from podocytes [10]. Second, there is evidence that allograft podocytes are produced from turnover of the recipient's bone marrow-derived cells [11]. Third, experimental evidence has shown that the transplantation of wild-type bone marrow into irradiated Alport mice results in the recruitment of podocytes, which produce type IV collagen $\alpha 5$ within the damaged glomerulus [12, 13]. We hypothesized that recurrence of AS occurs locally and influences the clinical abnormalities of patients with AS.

To address these clinical and histopathological issues, we investigated the graft survival and histopathological changes in patients with AS as well as the expression of GBM type IV collagen $\alpha 5$ and electron microscopy findings in renal allograft recipients with AS in the Japanese population.

\section{Methods}

We used the clinical, laboratory, and pathological data from recipients who underwent kidney transplantation at the Department of Urology, Tokyo Women's Medical University, and the Division of Nephrology and Hypertension, Department of Internal Medicine, The Jikei University School of Medicine, from February 1984 to February 2015. Twenty-one recipients had AS as the primary renal disease leading to end-stage renal disease. Controls included patients who underwent transplantation with end-stage renal disease due to a condition other than AS. The two groups of patients were matched for recipient and donor age, number of renal transplants, presence of donor-specific antibodies, donor resources, and era of transplantation using propensity-score analysis. Endopoints were graft loss or patients death.

\section{Immunosuppressive regimens and desensitization protocols}

All of the recipients were administered a triple immunosuppressive protocol comprising calcineurin inhibitors (CNI), antimetabolite drugs, and methylprednisolone (MP). Patients transplanted between 1989 and 1997 received cyclosporine and azathioprine (AZA), those transplanted between 1998 and 2000 received tacrolimus (TAC) and AZA, and those transplanted after 2001 received TAC and mycophenolate mofetil (MMF). After 2002, all patients received basiliximab perioperativelly. Splenectomy was performed at the time of transplantation between 1989 and 2004, and thereafter as an alternative to splenectomy, one dose of rituximab was administered 5-7 days before transplantation [14].

\section{Histopathology}

Two independent observers performed histological evaluation of formalin-fixed paraffin sections stained with hematoxylin and eosin, periodic acid-Schiff, Masson's trichrome, and periodic acid methenamine. All biopsies were evaluated based on the Banff 2013 classification.

\section{Immunofluorescence}

Immunohistochemistry was performed on cold acetone-fixed frozen sections using fluorescein isothiocyanate-conjugated anti- $\alpha 5$ (IV) (H53 and B51) and Texas red-conjugated anti- $\alpha 2$ (IV) (H51) (Shigei Medical Research Institute, Okayama, Japan). The immunoreactivities of type IV collagen $\alpha 2$ and $\alpha 5$ were observed and photographed by fluorescence microscopy (DP-70; Olympus, Tokyo, Japan). To evaluate histological recurrence of AS, we selected all recipients who underwent allograft biopsy more than one year after kidney transplantation. We could not obtain frozen biopsy specimens from some of those patients because the biopsy performed long before. As a result, we could assess frozen specimens obtained from six recipients. We evaluated two to three glomeruli per patient to identify focal or diffuse defects of type IV collagen $\alpha 5$ immunoreactivities in the GBM.

\section{Electron microscopy}

We observed the electron microscopic structure of the GBM in six patients of whom we could survey the allograft specimens in the long term more than one year after transplantation (Case1, 11, 12, 15, 16, 19). Among them we evaluated the electron microscopic figure of the GBM in two patients of whom we could survey the allograft specimens in particularly long term (101 and 110 months after kidney transplantation). For each, a 
total of 10 GBM measurements were evaluated on 10 randomly selected glomerular capillaries to determine the average (arithmetic mean) GBM thickness. Each electron micrograph was reviewed to determine whether foci of splitting or lamellation of the GBM was present.

\section{Biopsy and definition of AS recurrence}

Graft biopsy was performed based on episodic hematuria and proteinuria or worsening renal function. The other protocol biopsies were performed at 3 months, 1 year, and 3 years after kidney transplantation. We defined the recurrence of AS as either focal or diffuse defects of immunoreactivities of type IV collagen $\alpha 5$ chains in the GBM. On electron microscopy, we defined the recurrence of AS as the significant progression of irregular thinning and thickening of the GBM with splitting and lamellation of the lamina densa.

\section{Statistical analyses}

The baseline characteristics are presented as median with range or mean with standard deviation. We used Fisher's exact test to compare categorical variables between groups and the Mann-Whitney test to compare continuous variables. Allograft and patient survival were evaluated using the Kaplan-Meier method and compared between groups using the log-rank test. A two-tailed $p$-value of $<0.05$ was considered statistically significant.

\section{Results}

\section{Patient characteristics}

The characteristics of patients with AS and without AS are listed in Table 1. None of the variables except donor sex was significantly different between the two groups. The median age of the recipients was not significantly different between the AS and control groups [22 (range, 15-56) and 23 (range, 9-71) years, respectively; $p=$ 0.94]. The median follow-up period was 99.5 months (range, 0-348 months) and was not significantly different between the two groups $(p=0.78)$. A total of $71.4 \%$ $(15 / 21)$ of the patients in the AS group and 65.9\% (27/ 41) in the control group were male. Most patients underwent their first kidney transplantation (AS vs. control, $95.2 \%$ vs. $97.5 \%$, respectively), and the rates of LRKTx were not significantly different between the two groups (90.5\% in AS group vs. $92.7 \%$ in control group, $p$ $=1.0$ ). In our institutes, LRKTx occupy large majority of kidney transplantation. Among them, a few deceased donor kidney transplantation (DDKTx) were performed, which were $2 / 21(9.5 \%)$ and 3/41(7.3\%) in each Alport group and control group. Average age of recipient and donor were $29 / 20$ and 53/28 respectively in each group (Alport group/control group). In DDKTx cases, number of renal transplants, presence of donor-specific antibodies, and era of transplantation were comparable in
Table 1 Baseline characteristics in AS and control groups

\begin{tabular}{|c|c|c|c|}
\hline Variables & Alport group & Matched Control group & $p$ value \\
\hline $\mathrm{N}$ & 21 & 41 & \\
\hline Recipient age & $22(15-56)$ & $23(9-71)$ & 0.94 \\
\hline $\begin{array}{l}\text { Recipient gender } \\
\text { (Male) }\end{array}$ & $15(71.4 \%)$ & $27(65.9 \%)$ & 0.78 \\
\hline Recipient BMI & $18.3(14.4-22.9)$ & $20.2(14.6-27.2)$ & 0.083 \\
\hline $\begin{array}{l}\text { Time on Dialysis } \\
\text { (month) }\end{array}$ & $12(0-193)$ & $25(0-137)$ & 0.070 \\
\hline RRT modality (HD) & 17 (81.0\%) & $33(80.5 \%)$ & 1.0 \\
\hline Donor age & $52(40-65)$ & $50(16-78)$ & 0.42 \\
\hline Donor gender (Male) & $15(71.4 \%)$ & $15(37.5 \%)$ & $0.015^{*}$ \\
\hline \multicolumn{4}{|l|}{ No. of Transplantation } \\
\hline first & $20(95.2 \%)$ & $40(97.5 \%)$ & 1.00 \\
\hline second & $1(4.8 \%)$ & $1(2.4 \%)$ & NA \\
\hline third & $0(0.0 \%)$ & $0(0.0 \%)$ & \\
\hline \multicolumn{4}{|l|}{ HLA-mismatch } \\
\hline Class I & $1.33 \pm 0.73$ & $1.46 \pm 0.71$ & 0.46 \\
\hline Class II & $0.62 \pm 0.59$ & $0.78 \pm 0.42$ & 0.17 \\
\hline ABO incompatible & $5(23.8 \%)$ & $9(22.0 \%)$ & 1.0 \\
\hline Graft weight & $170(125-280)$ & $175(120-270)$ & 0.50 \\
\hline WIT (min) & $5(0-15)$ & $5(0-24)$ & 0.67 \\
\hline TIT (min) & $73(39-660)$ & $67.5(44-2233)$ & 0.55 \\
\hline \multicolumn{4}{|l|}{ Preformed DSA } \\
\hline HLA-Class I & $0 / 9(0.0 \%)$ & $5 / 21(23.8 \%)$ & 0.30 \\
\hline HLA-Class ॥ & $0 / 6(0.0 \%)$ & 3/19 (15.8\%) & 1.0 \\
\hline Graft failure & $6(28.6 \%)$ & 19 (45.2\%) & 0.27 \\
\hline Chronic rejection & 4 & 14 & 0.89 \\
\hline Non-compliance & 1 & 1 & 0.073 \\
\hline Unknown & 1 & 4 & NA \\
\hline $\begin{array}{l}\text { Follow up period } \\
\text { (month) }\end{array}$ & $83(5-315)$ & $110(0-348)$ & 0.78 \\
\hline Living donor RTx & 19 (90.5\%) & $38(92.7 \%)$ & 1.0 \\
\hline \multicolumn{4}{|l|}{ Era of transplantation } \\
\hline-1989 & $3(14.3 \%)$ & $5(12.2 \%)$ & 1.0 \\
\hline 1990-1999 & $4(19.0 \%)$ & $11(26.8 \%)$ & 0.55 \\
\hline 2000-2009 & $6(28.5 \%)$ & $18(43.9 \%)$ & 0.28 \\
\hline $2010-$ & $8(38.1 \%)$ & $7(17.1 \%)$ & 0.12 \\
\hline Hematuria $^{\text {b }}$ & $2 / 18(11.1 \%)$ & $5 / 25(20.0 \%)$ & 0.69 \\
\hline
\end{tabular}

DSA: donor-specific antibody, RTx: renal transplantation, TCMR: T cell-mediated rejection, ABMR: Antibody-mediated rejection

apreformed DSA was evaluated by HLA-antibody single antigen test (Luminex method). This test for HLA-Class I was performed to nine cases in AS and twenty-one cases in control, and for HLA-Class II to six cases in AS and nineteen cases in control

${ }^{\mathrm{b}}$ Hematuria displays the number of recipients who had hematuria more than $(1+)$ at the latest examination/total recipients who could be obtained the information of latest urine examination ${ }^{*} p$-value was $<0.05$ 
both groups, without any significant difference. Human leukocyte antigen mismatch, ABO compatibility, preformed donor-specific antibody, and the era of kidney transplantation were not significantly different between the two groups. The primary disease in 10 patients $(24.4 \%)$ in the control group $(N=41)$ was predominantly reflux nephropathy, followed by IgA nephropathy in 5 patients $(12.2 \%)$, focal segmental glomerulosclerosis in 3 (7.3\%), diabetic nephropathy in $1(2.4 \%)$, other conditions in 12 (29.3\%), and unknown in 6 (14.6\%) (Table 2). Fifteen of 21 recipients were male, and the donors comprised 10 fathers, 3 mothers, and 2 cadavers in AS group. Six female recipients underwent donations from three fathers, two mothers, and one husband (Table 3). We could obtain the information of hematuria in 43 recipients $(69.3 \%$, Alport group: 18cases, matched control group: 25 cases) at the latest urine sample. Only few patients showed positive hematuria at the latest urine examination. There was no statistical difference in patients who showed positive hematuria between Alport group (2/18: $11.1 \%)$ and matched control group (5/25: $20.0 \%)$. We investigated the episodes of rejection in Alport group and control group. The rate of cases who underwent rejection were 8/17 (47.1\%), 20/33 (60.6\%) in Alport group and Control group, respectively. The results were not significantly different in both groups.

\section{Patients and graft survival in AS and matched control groups}

The cumulative patient survival rate was not significantly different between the two groups $(p=0.11$, log-rank test) (Fig. 1). The 10-year patient survival rate after transplantation was $100 \%$ in the AS group and $94.6 \%$ in the matched control group. The cumulative graft survival rate was higher in the AS group (100\%, $86.7 \%$, and $69.3 \%$ at 1,5 , and 10 years, respectively) than that in the control group $(95.1 \%, 77.1 \%$, and $64.2 \%$ at 1 , 5 , and 10 years, respectively), but the difference was not significant ( $p=0.16, \log$-rank test) (Fig 2). Five patients (28.6\%) in the AS group and 19 (45.2\%) in the matched control group developed allograft failure mainly caused

Table 2 Primary disease in control group

\begin{tabular}{ll}
\hline Primary disease & Number (\%) \\
\hline Reflux nephropathy & $10(24.4 \%)$ \\
IgA nephropathy & $5(12.2 \%)$ \\
FSGS & $3(7.3 \%)$ \\
Hypoplastic kidney & $2(4.9 \%)$ \\
RPGN & $2(4.9 \%)$ \\
Diabetic nephropathy & $1(2.4 \%)$ \\
Others & $12(29.3 \%)$ \\
Unknown & $6(14.6 \%)$ \\
\hline
\end{tabular}

by chronic rejection $(66.7 \%$ in $\mathrm{AS}$ group vs. $73.7 \%$ in control group) or by non-compliance $(16.0 \%$ in AS group vs. $5.3 \%$ in control group). There were no cases of recurrent nephropathy in the AS group.

\section{Long-term immunoreactivity of type IV collagen in GBM of AS allograft specimens after kidney transplantation} We evaluated the graft specimens in 6 of 21 patients in the AS group who underwent allograft biopsy more than 1 year after kidney transplantation (Table 3 ). The median age of these patients at the time of kidney transplantation was 24 years (range, 21-27 years), and five of the six patients were male. The median duration of time from kidney transplantation to allograft biopsy was 23.5 months (range, 12-110 months). Type IV collagen $\alpha 5$ chains in the GBM of all patients were maintained linearly, and even focal depletion was not observed (Fig. 3). In one patient with chronic active antibody-mediated rejection, the $\alpha 2$ chains showed thickening in the GBM that extended to subendothelial lesions (Fig. 3, Case 12).

\section{Long-term electron microscopic findings of GBM in allograft specimens of AS recipients after kidney transplantation}

We assessed the electron microscopic findings of six patients who underwent allograft biopsy. Cases 11 and 16 (data not shown) showed a focally thin basement membrane in the GBM, suggesting the transmission of thin basement membrane disease. Among these patients, we evaluated detailed long-term data after kidney transplantation (101 and 110 months after transplantation in Cases 11 and 12, respectively). We found no significant changes, such as splitting or lamination of the GBM, in both cases. Case 11 showed a thickness of $275 \mathrm{~nm}$ at 101 months after kidney transplantation $339 \mathrm{~nm}$ at $0 \mathrm{~h}$. Case 12 showed a thickness of $220 \mathrm{~nm}$ at 110 months after transplantation and $146 \mathrm{~nm}$ at $0 \mathrm{~h}$, suggesting the transmission of thin basement membrane disease. This case was characterized by chronic antibody-mediated rejection by light microscopy and showed a double-countered GBM with extended edema of the subendothelial lesion and fusion of foot process, suggesting endothelial and podocyte injury.

\section{Discussion}

This study is the first to demonstrate patient and graft survival in the Japanese population of patients with AS. We retrospectively reviewed the long-term outcomes of 21 patients with AS who underwent kidney transplantation with a mean follow-up duration of 83 months and found that the cumulative patient and graft survival rates in the AS group were not significantly different from those of a well-matched control group (Figs. 1 and 2). Our data as well as those reported by Yilmaz from 


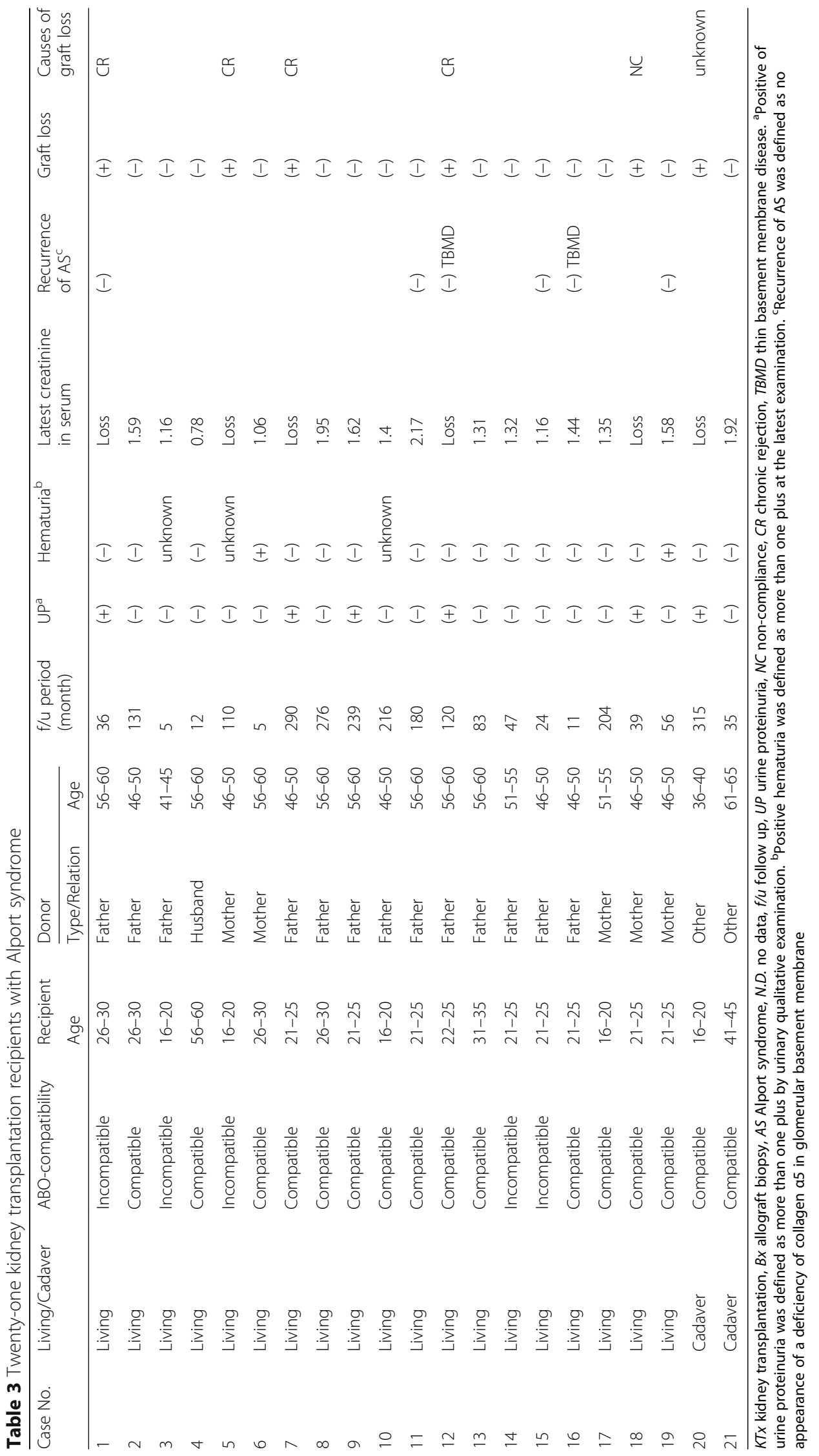




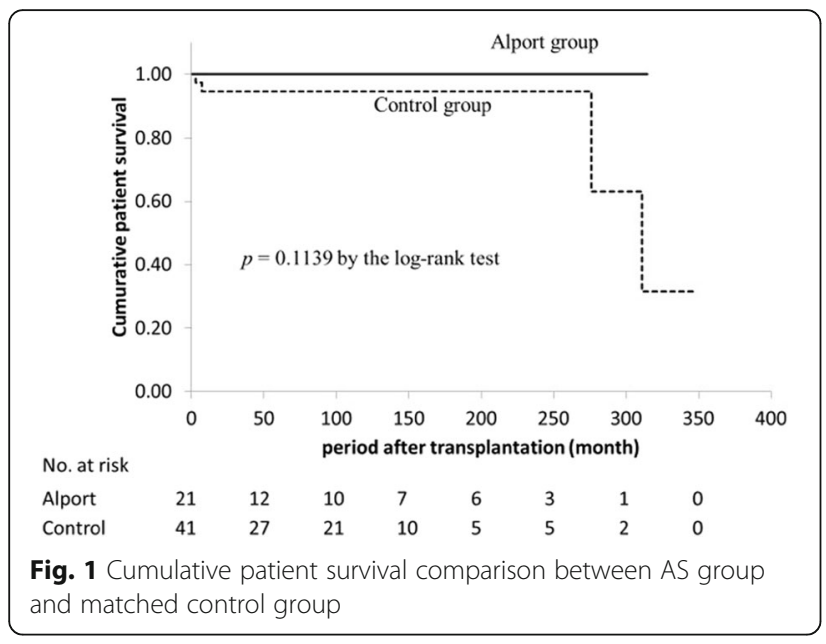

Turkey showed no difference between patients with and without AS [15]. In contrast, Temme showed better graft survival (HR, 0.75; 95\% CI, 0.60-0.93; $p=0.008)$ and patient survival (HR, 0.46; 95\% CI, 0.30-0.59; $p=0.001$ ) in patients with AS using the ERA-EDTA Registry data [7]. The only difference in the patients' characteristics was the rate of deceased donors $(63.0 \%$ in the ERA-EDTA data, $32.0 \%$ in Yilma's data, and $9.5 \%$ in our data). Notably, the author demonstrated that the patient and graft survival rates were better in deceased donors than in controls, but not in living donors. This finding suggests that living donors with AS could negatively affect patient and graft survival. In line with this, Gross from Germany observed six cases of living donor kidney transplantation in AS from relatives with mild urinary abnormalities and found that three of the six donors developed new-onset hypertension and two of the six donors developed new-onset proteinuria. The author pointed out that, genetically, AS may also affect other family members, and physicians should be aware of an increased risk of renal

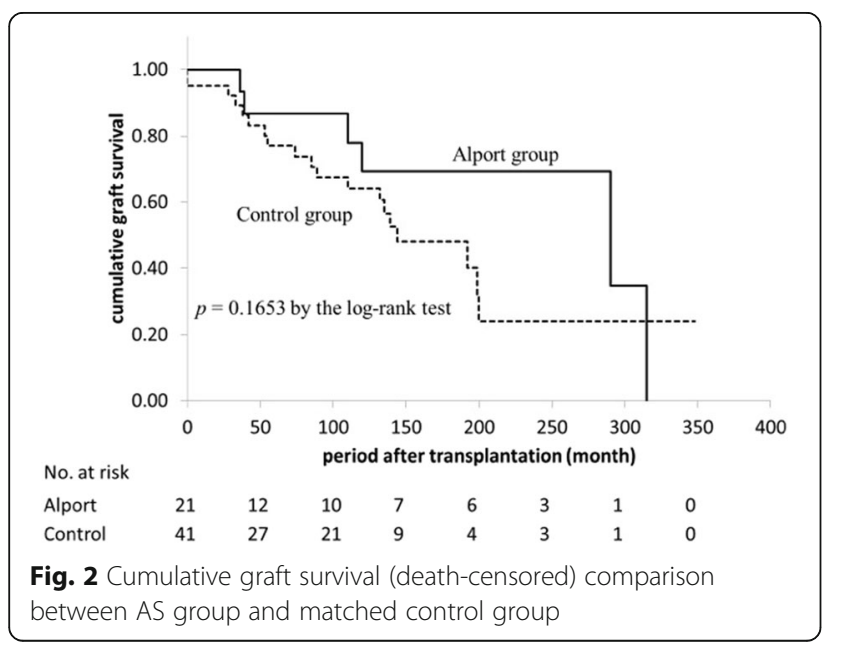

failure in both the donor and the recipient [16]. In this context, in countries with a donor shortage, such as Japan (in which almost $90 \%$ of transplantations are living donor kidney transplantations), careful evaluation and monitoring of both donors and recipients with AS are necessary. In the present study, 13 of 21 patients received kidneys from a father, suggesting that most donations were from an unaffected father with X-linked (COL4A5 gene) AS. However, we found at least two patients (Cases 12 and 16) with a relatively thin GBM on electron microscopy, suggesting possible transmission of thin basement membrane disease from autosomal-recessive (COL4A3 or COL4A4 gene) affected relatives $[17,18]$. For example, in Case 16, we confirmed that type IV collagen $\alpha 5$ staining of the recipient's native kidney biopsy revealed positive in the basement membrane of Bowman's capsule but negative in GBM, suggesting autosomal-recessive AS associated with COL4A3/4 gene abnormality. Of note, previous report showed that about $40 \%$ of TBMD patients have heterozygous mutations at the COL4A3/4 locus [19]. Therefore, the donor in Case 16 could be heterozygous carrier with autosomal-recessive AS, resulting in TBMD shown in baseline allograft biopsy. These two patients had stable kidney function and did not show proteinuria or hematuria. These results suggest that living donor kidney transplantation from relatives with AS is acceptable if the donors and recipients are carefully evaluated. Another important factor associated with patient and graft outcomes is the occurrence of anti-GBM disease. Anti-GBM disease induces graft failure in the early stage of kidney transplantation in recipients with AS, but the incidence is generally very low at $1.9 \%$ [9]. Fortunately, no recipients in the AS group developed anti-GBM disease within the observation. Among patients with AS, the risk factors associated with graft loss in patients other than living donors and those with anti-GBM disease have not been fully evaluated. In the present study, 6 of 21 (28.6\%) recipients with AS developed graft loss. Of these, four (66.6\%) were due to chronic rejection, one (16.7\%) was due to noncompliance with medication, and another was of unknown cause. These results are comparable with the causes of graft loss in the matched control group.

Another main finding of this study is that no patients developed recurrence of AS. To the best of our knowledge, this is the first study to evaluate the recurrence of AS in detail using histopathological methods. Generally, recurrent glomerulonephritis or glomerulopathy may cause graft loss in kidney transplantation, but most nephrologists believe that patients with AS do not develop recurrence. However, several recent clinical and experimental studies have implied that GBM type IV collagen originates from podocytes recruited from the recipient's bone marrow-derived cells, suggesting the possibility of recurrence of AS. For example, Abrahamson clearly demonstrated that 


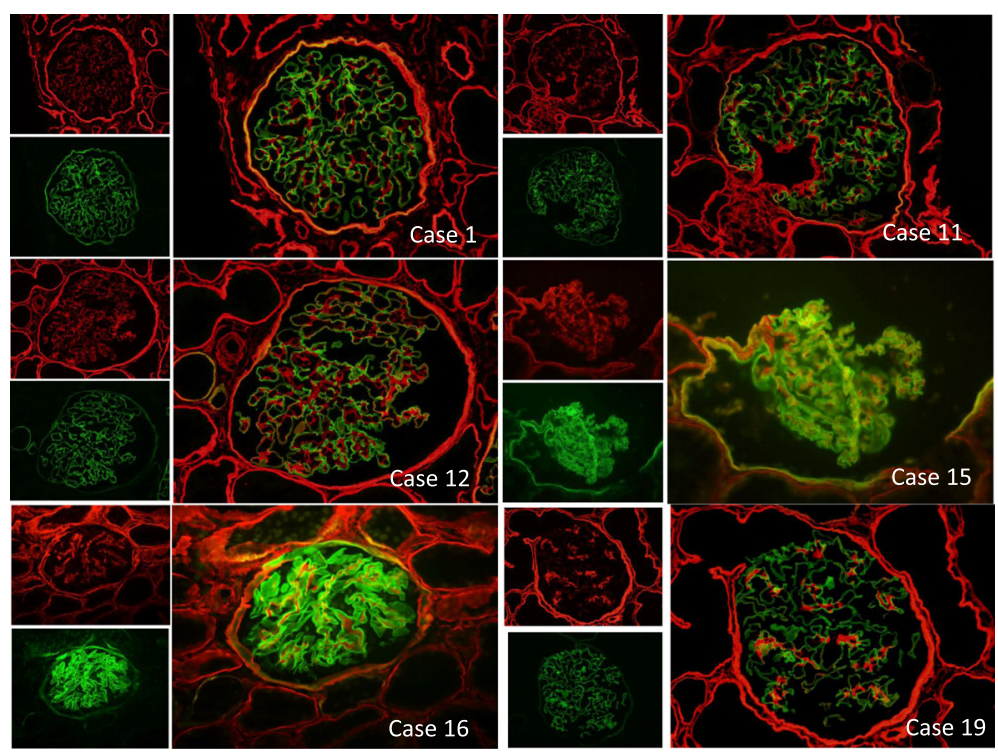

Fig. 3 Immunofluorescence staining of type IV collagen a5 chain. Immunofluorescence staining of type IV collagen a2 chain (red), a5 chain (green), and merged images (combined red and green) of GBM on allograft biopsy specimens of AS recipients performed more than 1 year after transplantation (patient characteristics are shown in Table 3). In all cases, the type IV collagen a5 chain was stained linearly in the GBM without defects. Case1: A 27-yearold woman. Allograft biopsy specimen at 33 months after transplantation. The pathological diagnosis was chronic active antibody-mediated rejection. Case 11: A 21-year-old man. Allograft biopsy specimen at 101 months after transplantation. The pathological diagnosis was arteriolar hyalinosis. Case 12: A 21year-old man. Allograft biopsy specimen at 110 months after transplantation. The pathological diagnoses were chronic active antibody-mediated rejection and IF/TA, moderate. The immunoreactivity of type IV collagen a2 chain was slightly increased in the mesangial and subendothelial regions compared with that in the other cases. Case 15: A 26-year-old man. Allograft biopsy specimen at 12 months after transplantation. The pathological diagnosis was minimally aggressive tubulointerstitial rejection, mild. Case 16: A 26-year-old man. Allograft biopsy specimen at 12 months after transplantation. The pathological diagnosis was no evidence of rejection. Case 19: A 23-year-old man. Allograft biopsy specimen at 14 months after transplantation. The pathological diagnosis was IF/TA, mild

$\alpha 3 \alpha 4 \alpha 5$ chains are produced only by podocytes, as evidenced by immunoelectron microscopic examination [10]. In human kidney transplantation research, Becker showed that recipient-derived podocytes were found in 4 of 8 biopsies, representing 3 of 6 patients, and that 5 of the 740 podocytes examined in the female-donated allograft were male-derived, using fluorescence in situ hybridization for the $\mathrm{X}$ and $\mathrm{Y}$ chromosomes [11]. In addition, Sugimoto et al. reported that bone marrow transplantation from wild-type mice to Alport model mice (COL4A3 -/-) improved the symptoms of AS and the level of protein produced from bone marrow-derived cells [12]. Therefore, we initially hypothesized that the podocytes of allografts can be replaced by recipient-derived podocytes that cannot synthesize $\alpha 3 \alpha 4 \alpha 5$ chains, resulting in deficiency of the GBM of allograft. However, we found that the structure of the GBM remained normal and that no deficiencies of the $\alpha 5$ chains in the GBM occurred, even long after kidney transplantation, suggesting that AS does not recur histologically. We speculated following two mechanisms for this phenomenon:1) a very small number of donor derived podocytes were replaced by recipient derived ones [11], or 2) podocytes injury is rare in recipients with AS, and both podocytes and
GBM turnover was not evident [20-22]. Additionally, only few recipients in Alport group showed hematuria in the latest examination (11.1\%, Table 1), which further strengthen no histological recurrence of AS because electron microscopy might underestimate the presence of GBM changes of AS due to sampling bias.

The strengths of this study include the selection of controls (propensity-score analysis with matching of six factors: recipient and donor age, number of transplants, donor-specific antibody positivity, donor resources, and era of transplantation) and longer observational periods than in previous reports (99.5 vs. 75.4 months) [15]. Limitations include the retrospective design, small sample size due to the rarity of AS, lack of genetic information, and performance of few allograft biopsies because the allograft function was relatively good, even long after kidney transplantation, in recipients with AS.

\section{Conclusion}

Our data demonstrate that graft survival in patients with AS is similar to that in patients with other primary diseases in the Japanese population. Careful selection of living donors is necessary for the successful management of kidney transplantation in patients with AS. Neither 
deficiency of the $\alpha 5$ chains (type IV collagen) nor GBM abnormalities were detected in the kidney allograft biopsies, indicating that AS recurrence was not present or was very limited in recipients with AS. Further pathological analysis with a longer follow-up to evaluate the possible recurrence of AS and transmission of thin basement membrane disease from affected relatives will contribute to a better understanding of kidney transplantation in patients with AS.

\section{Abbreviations \\ ABMR: antibody-mediated rejection; AS: Alport syndrome; DDKTx: deceased donor kidney transplantation; GBM: glomerular basement membrane; IF/ TA: interstitial fibrosis and tubular atrophy; KTx: kidney transplantation; LRKTX: living related kidney transplantation}

\section{Acknowledgments}

We appreciate the support provided by Katsunori Shimada, PhD (STATZ Institute, Inc., Tokyo, Japan), who provided expert assistance with the statistical analysis.

\section{Ethical approval and consent to participate}

Approval was obtained from the Institutional Ethics Committee of Tokyo Women's Medical University (Identifier: 3336-R) and the Institutional Ethics Committee of The Jikei University School of Medicine [Identifier: 28-003(8246)]. Written informed consent was obtained from all selected patients.

\section{Funding}

Astellas Pharma Inc. (Tokyo, Japan) supported this study with a grant. The sponsor was not involved in the study design, patient enrollment, data collection, data analysis, data interpretation, or preparation of the manuscript.

\section{Availability of data and materials}

No data has been submitted to any open access databases. Full patient records are being kept coded.

\section{Author's contributions}

AK and YN designed this study and drafted the article. SH embedded, cut, and stained the renal biopsies. HK, MF, KU corrected the clinical data. IY proposed the research design and corrected the article. $\mathrm{MO}$ and $\mathrm{HI}$ provided those clinical data and closely examined the article. TY and KT closely examined and corrected the article. All the authors contributed to preparation of the manuscript. In addition, all authors have read and approved the final manuscript.

\section{Competing interest}

The authors declare that they have no competing interests.

\section{Consent for publication}

Written informed consents for the use of clinical data and publication purpose were obtained from all participants. Images are entirely unidentifiable and there are no details on individuals reported within the manuscript.

\section{Publisher's Note}

Springer Nature remains neutral with regard to jurisdictional claims in published maps and institutional affiliations.

\section{Author details}

'Division of Nephrology and Hypertension, Department of Internal Medicine, The Jikei University School of Medicine, 3-25-8, Nishi-Shimbashi, Minato-ku, Tokyo 105-8461, Japan. ²Department of Medicine, Kidney center, Tokyo Women's Medical University, Tokyo, Japan. ${ }^{3}$ Department of Urology, Tokyo Women's Medical University, Tokyo, Japan.
Received: 17 April 2017 Accepted: 20 September 2018

Published online: 03 October 2018

\section{References}

1. Hudson BG, Tryggvason K, Sundaramoorthy M, Neilson EG. Alport's syndrome, Goodpasture's syndrome, and type IV collagen. N Engl J Med. 2003:348(25):2543-56

2. Heidet L, Cai Y, Guicharnaud L, Antignac C, Gubler MC. Glomerular expression of type IV collagen chains in normal and X-linked Alport syndrome kidneys. Am J Pathol. 2000;156(6):1901-10.

3. Kruegel J, Rubel D, Gross O. Alport syndrome--insights from basic and clinical research. Nat Rev Nephrol. 2013;9(3):170-8.

4. Gross O, Licht C, Anders HJ, Hoppe B, Beck B, Tonshoff B, Hocker B, Wygoda S, Ehrich JH, Pape L, Konrad M, Rascher W, Dotsch J, Muller-Wiefel DE, Hoyer P, Study group members of the Gesellschaft fur Padiatrische Nephrologie, Knebelmann B, Pirson Y, Grunfeld JP, Niaudet P, Cochat P, Heidet L, Lebbah S, Torra R, Friede T, Lange K, Muller GA, Weber M. Early angiotensinconverting enzyme inhibition in Alport syndrome delays renal failure and improves life expectancy. Kidney Int. 2012;81(5):494-501.

5. Kashtan CE, Ding J, Gregory M, Gross O, Heidet L, Knebelmann B, Rheault M, Licht C, Collaborative ASR. Clinical practice recommendations for the treatment of Alport syndrome: a statement of the Alport syndrome research collaborative. Pediatr Nephrol. 2013;28(1):5-11.

6. Savige J, Gregory M, Gross O, Kashtan C, Ding J, Flinter F. Expert guidelines for the management of Alport syndrome and thin basement membrane nephropathy. J Am Soc Nephrol. 2013;24(3):364-75.

7. Temme J, Kramer A, Jager KJ, Lange K, Peters F, Muller GA, Kramar R, Heaf JG, Finne P, Palsson R, Reisaeter AV, Hoitsma AJ, Metcalfe W, Postorino M, Zurriaga O, Santos JP, Ravani P, Jarraya F, Verrina E, Dekker FW, Gross O. Outcomes of male patients with Alport syndrome undergoing renal replacement therapy. Clin J Am Soc Nephrol. 2012;7(12):1969-76.

8. Gross O, Kashtan CE, Rheault MN, Flinter F, Savige J, Miner JH, Torra R, Ars E, Deltas C, Savva I, Perin L, Renieri A, Ariani F, Mari F, Baigent C, Judge P, Knebelman B, Heidet L, Lagas S, Blatt D, Ding J, Zhang Y, Gale DP, Prunotto M, Xue Y, Schachter AD, Morton LC, Blem J, Huang M, Liu S, Vallee S, Renault D, Schifter J, Skelding J, Gear S, Friede T, Turner AN, Lennon R. Advances and unmet needs in genetic, basic and clinical science in Alport syndrome: report from the 2015 international workshop on Alport syndrome. Nephrol Dial Transplant. 2017:32(6):916-24.

9. Byrne MC, Budisavljevic MN, Fan Z, Self SE, Ploth DW. Renal transplant in patients with Alport's syndrome. Am J Kidney Dis. 2002:39(4):769-75.

10. Abrahamson DR, Hudson BG, Stroganova L, Borza DB, St John PL. Cellular origins of type IV collagen networks in developing glomeruli. J Am Soc Nephrol. 2009;20(7):1471-9.

11. Becker JU, Hoerning A, Schmid KW, Hoyer PF. Immigrating progenitor cells contribute to human podocyte turnover. Kidney Int. 2007;72(12):1468-73.

12. Sugimoto H, Mundel TM, Sund M, Xie L, Cosgrove D, Kalluri R. Bone-marrowderived stem cells repair basement membrane collagen defects and reverse genetic kidney disease. Proc Natl Acad Sci U S A. 2006;103(19):7321-6.

13. Prodromidi El, Poulsom R, Jeffery R, Roufosse CA, Pollard PJ, Pusey CD, Cook $H T$. Bone marrow-derived cells contribute to podocyte regeneration and amelioration of renal disease in a mouse model of Alport syndrome. Stem Cells. 2006;24(11):2448-55.

14. Okumi M, Toki D, Nozaki T, Shimizu T, Shirakawa H, Omoto K, Inui M, Ishida $H$, Tanabe K. ABO-incompatible living kidney transplants: evolution of outcomes and immunosuppressive management. Am J Transplant. 2016;16:886-96.

15. Yilmaz VT, Dinckan A, Yilmaz F, Suleymanlar G, Kocak H. Outcomes of renal transplantation in patients with Alport syndrome. Transplant Proc. 2015; 47(5):1377-81

16. Gross O, Weber M, Fries JW, Müller GA. Living donor kidney transplantation from relatives with mild urinary abnormalities in Alport syndrome: longterm risk, benefit and outcome. Nephrol Dial Transplant. 2009 May;24(5): $1626-30$.

17. Ivanyi B, Pap R, Ondrik Z. Thin basement membrane nephropathy: diffuse and segmental types. Arch Pathol Lab Med. 2006 Oct;130(10):1533-7.

18. Pierides A, Voskarides K, Athanasiou Y, loannou K, Damianou L, Arsali M, Zavros M, Pierides M, Vargemezis V, Patsias C, Zouvani I, Elia A, Kyriacou K, Deltas C. Clinico-pathological correlations in 127 patients in 11 large pedigrees, segregating one of three heterozygous mutations in the COL4A3/ COL4A4 genes associated with familial haematuria and significant 
late progression to proteinuria and chronic kidney disease from focal segmental glomerulosclerosis. Nephrol Dial Transplant. 2009;24(9):2721-9.

19. Haas M. Alport syndrome and thin glomerular basement membrane nephropathy: a practical approach to diagnosis. Arch Pathol Lab Med. 2009 Feb;133(2):224-32.

20. Wanner N, Hartleben B, Herbach N, Goedel M, Stickel N, Zeiser R, Walz G, Moeller MJ, Grahammer F, Huber TB. Unraveling the role of podocyte turnover in glomerular aging and injury. J Am Soc Nephrol. 2014;25:707-16.

21. Price RG, Spiro RG. Studies on the metabolism of the renal glomerular basement membrane. Turnover measurements in the rat with the use of radiolabeled amino acids. J Biol Chem. 1977;252:8597-602.

22. Walker $F$. The origin, turnover and removal of glomerular basementmembrane. J Pathol. 1973;110:233-44.

Ready to submit your research? Choose BMC and benefit from:

- fast, convenient online submission

- thorough peer review by experienced researchers in your field

- rapid publication on acceptance

- support for research data, including large and complex data types

- gold Open Access which fosters wider collaboration and increased citations

- maximum visibility for your research: over $100 \mathrm{M}$ website views per year

At BMC, research is always in progress.

Learn more biomedcentral.com/submissions 